\title{
INTERACTIONS SOCIALES CHEZ LES POISSONS : LES PHEROMONES
}

Ph. SAGLIO

I.N.R.A., Laboratoire de Physiologie des Poissons 78350 JOUY EN JOSAS, France

1. Position du problème et définition des phéromones

2. Rôle des phéromones dans les différents aspects du phénomène social
A) Comportement sexuel
B) Orientation et * homing *
C) Relations parents-jeunes
D) Reconnaissance intraspécifique et comportement de banc
- Réaction d'alarme
- Effet de groupe

3. Conclusions et perspectives d'avenir

\footnotetext{
- Exposé présenté lors du séminaire I.N.R.A. - C.S.P., Grignon, Février 1979
} 


\section{I. - POSITION DU PROBLEME ET DEFINITION DES PHEROMONES}

Après s'être longtemps consacrèes à l'étude presque exclusive de la vision. les recherches er: psychologie sensorielle se sont attachées plus particulièrement depuis une trentaine d'années à déterminer le rôle joué par le sens chimique (olfaction et gustation essentiellement) dans les comportements fondamentaux des animaux.

Parmi les vertébrés non mamaliens, les Poissons représentent la superclasse chez laquelle les problèmes liès à la chémoréception ont été les plus ètuoies. Selon la définition de KARLSON et LUSCHER (1959) les phèromones désignent les substances qui, sécrétées à l'extérieur par un individu, provoquent chez un individu de la même espèce un comportement déterminé ou un processus de développement physiologique. Il est utile de rappeler à l'occasion la distinction effectuée entre ces médiateurs chimiques possèdant une action interindividuelle intraspécifique; les hormones, médiateurs chimiques agissant au niveau mème de l'organisme responsable de leur sécrétion et les substances allélochimiques, qui présentent une action interindividuelle interspécifique.

Nous nous limiterons ici à présenter le rôle joué par les phéromones dans les différents aspects du phénomène social chez les Poissons.

\section{II. - ROLE DES PHEROMONES DANS LES DIFFERENTS ASPECTS DU PHENOMENE SOCIAL}

\section{A) Comportement sexuel}

Malgré les travaux de TAVOLGA qui montraient en 1956 que l"excitation sexuelle du mâle de Bathygobius soporator (Gobiidé) ètait due à la perception d'une phéromone émise par la femelle ovulée, les recherches sur les phéromones sexuelles de Poissons se sont développées très tardivement. Ceci expilque en partie pourquoi FRINGS et FRINGS (1964) pouvaient encore affirmer il y a une quinzaine d'années que, parmi les vertébrés, seuls les mammifères utilisaient leur sens olfactif dans la communication sexuelle. Depuis, l'intervention de phéromones sexuelles dans la reproduction a été mise en évidence chez de nombreuses espèces de Salmonidés, de Siluridés, de Cyprinidés et de Blenniidés.

Utilisant un olfactomètre en $Y$, NEWCOMBE et HARTMAN montraient en 1973 que des truites arc-en-ciel Salmo gairdneri mâles et femelles, sexuellement matures, étaient très attirées par l'eau provenant de poissons de la même espèce frayant en amont. L'an passé, EMANUEL et DODSON ont prouvé que l'orientation et la cinétique de la réaction rhéotropique de mâles de cette espèce soumis à un courant d'eau chargé de fluide ovarien étaient sous contrôle olfactif.

Chez lctalurus nebulosus, TIMMS et KLEEREKOPER (1972) démontraient la nature chimique de la perception de la femelle gravide par le mâle à maturité sexuelle et le caractère tropotaxique du mécanisme d'orientation présenté par ce dernier. Cette particularité est d'ailleurs mise à profit par certains pêcheurs du Mississipi qui ont coutume d'utiliser des femelles en période reproductrice comme leurres pour capturer dans leurs nasses un grand nombre de mâles spécifiques. 
Après réalisation et test d'extraits de différentes origines (épiderme, appareil digestif et ovaire) AMOURIQ concluait en 1965 que l'hyperactivité induite chez le mâle de Guppy Poecilia reticulata par la femelle spécifique était due à une phéromone d'origine ovarienne que l'auteur qualifiait de * substance dynamogène". Chez cette même espèce, CROW et LILEY (1979) ont montré tout iécemment que les mâles "préfèrent" l'eau qui a contenu des femelles durant 'es premiers jours du cycle reproducteur à celle qui a contenu des femelles au milieu de leur cycle ou des femelles qui ont subi l'ablation des ovaires. Ces auteurs constatent que l'intensité de la cour faite par les mâles aux femelles ovariectomisées augmente considérablement lorsque l'on ajoute de l'eau ayant contenu des femelles intactes.

Chez le poisson rouge, Carassius auratus, PARTRIDGE, LILEY et STACEY (:976) concluent également de leurs expériences que, peu après l'ovulation, la femelle emet une phéromone d'origine ovarienne évacuée au niveau du pore génital déclenchant l'attraction et le comportement de poursuite caractéristique ¿u mâ'e spermiant. L'anosmie du mâle par occlusion des narines ou section des nerfs olfactifs supprime presque totalement la réaction, ceci suggérant le rôle essentiel joué par l'olfaction dans la reproduction de cette espèce.

Il existe enfin des espèces chez lesquelles la reproduction nécessite la perception par la femelle d'une phéromone émise par le mâle. C'est le cas de Brachydanio rerio CCHEN et MARTINICH, 1975) et de Blennius pavo (LAUMEN et BLUM, 1973). Chez cette dernière espèce, les auteurs ont montré que les structures glandulaires responsables de l'émission de la phéromone du mâle se trouvaient au niveau de la nageoire anale et se présentaient sous la forme d'appendices plus ou moins développés selon le stade physiologique sexuel. L'injection de LH hypophysaire d'origine mammalienne, à des mâles immatures, provoque le développement fonctionnel de ces appendices et l'attraction des femelles.

\section{B) Orientation et "homing "}

Les phèromones semblent jouer un rôle déterminant chez les poissons migrateurs, provoquant chez ceux-ci un comportement d'aggrégation spécifique à certaines périodes de leur vie. GERKING (1959) utilisait le terme de "homing" pour qualifier le retour d'un poisson après une migration, un déplacement accidentel ou expérimental, à un endroit bien défini. Dès 1880, BUCKLAND suggérait que le saumon adulte était capable de reconnaitre les caractéristiques chimiques de la rivière natale. En 1934, après implantation de fraie de saumon atlantique, Salmo salar, dans une rivière connue comme ne possédant aucun poisson de cette espèce. WHITE put y noter une importante remontée de saumons dans l'année qui suivit.

L'hypothèse olfactive proposee par HASLER et WISBY (1951) impliquait rue :

- le cours d'eau possède une odeur spécifique perceptible par le poisson :

- le poisson soit capable de discriminer entre les odeurs de différents cours d'eau;

- le poisson puisse conserver "l'engramme olfactif" caractéristique.

Les différentes études de transplantation d'œufs et d'alevins de saumons atlantique ou de coho (Oncorhynchus kisutch) (CARLIN, 1959-1968; DONALDSON et ALLEN. 1957; PECK, 1970; JENSEN et DUNCAN, 1971) ont montré que la 
connaissance des caractéristiques du cours d'eau repose essentiellement sur un processus d'engrammation réalisé au moment où le juvénile commence sa migration aval.

Afin de déterminer chez les Salmonidés l'importance du rôle joué par l'olfaction dans le choix de la rivière et le retour au site de fraie. HASLER et WISBY (1951) proposèrent d' "imprégner "les poissons, de l'œuf au stade * smolt " à un composé chimique n'existant pas dans la nature, ne possédant pas d'activité attractive ou répulsive, très soluble dans l'eau et perceptible à faible concentration.

12 à 18 mois environ après le lâcher initial des smolts marqués, le composé serait introduit dans la rivière expérimentale et l'efficacité de l'imprégnation olfactive serait apprécié grâce au nombre de poissons marqués capturés

La « morpholine $*$, perçue à une concentration aussi faible que $5.10^{-5} \mathrm{mg} / \mathrm{l}$ fut le composé retenu.

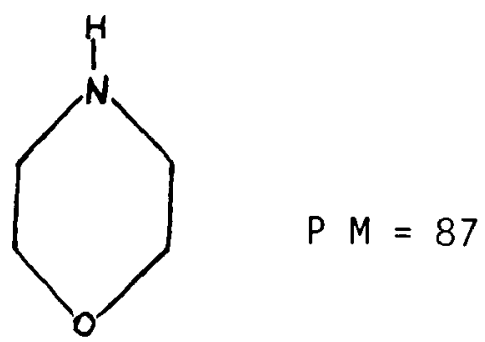

\section{Morpholine}

Les nombreuses expériences d'imprégnation artificielle à ce composé réalisées ces dernières années aux Etats-Unis et au Canada sur le saumon coho et la truite arc-en-ciel (SCHOLZ et al., 1975; COOPER et al., 1976; MADISON et al. 1973) ont prouvé l'efficacité de la méthode, les taux de recapture étant dans tous les cas hautement significatifs.

Nous dresserons toutefois quelques critiques concernánt l'utilisation de la morpholine dans l'induction du homing chez les Salmonidés

D'après les enregistrements électrophysiologiques réalisés par HARA (1974), la stimulation du bulbe olfactif de la truite arc-en-ciel avec une solution de morpholine à $1 \%$ produit une réponse différente de la réponse normale du bulbe olfactif et inhibe la sensibilité olfactive à d'autres composés. Selon cet auteur, le mécanisme responsable de l'imprégnation à la morpholine n'est pas directement associé à la fonction olfactive. Ainsi, on introduit dans l'environnement de poissons expérimentaux (ou non) une substance synthétique dont on ignore la nature exacte de l'effet psychophysiologique, tout en risquant de perturber les capacités de perception olfactive à d'autres composés tels que des acides aminés qui, cela a été montré (IDLER et al., 1956; HARA, 1974; HARA et al., 1973) jouent un rôle essentiel dans la vie des salmonidés.

Enfin, l'utilité même de la méthode demeure discutable dans la mesure où des expériences, relativement plus simples, de déplacements simultanés de poissons marqués témoins et de poissons marqués porteurs de lésions olfactives ou (et) visuelles prouvent, sans ambiguïté, à la fois la possibilité d'implantation 
d'une nouvelle population de salmonidés et le rôle prépondérant joué par l'olfaction dans le phénomene de "homing *. Outre les salmonidés, les observations de DODSON et LEGGET (1974) sur les déplacements de l'Alose savoureuse, Alosa sapidissima, comme celles de MILES (1968) concernant ceux de l'anguille américaine. Anguilla rostrata, s'accordent également pour donner une place capitale à l'olfaction.

\section{C) Relations parents-jeunes}

Chez certaines espèces de poissons, l'identification et le soin des jeunes par les parents s'effectuent sous contrôle chèmosensoriel. Ceci a été bien mis en évidence chez des représentants de la famille des Cichlidés (NOBLE et CURTIS, 1939; KUHME, 1963 ; GREENBERG, 1963 ; KUNZER, 1964 ; MYRBERG. 1966), famille caracterisèe par le haut niveau de complexité des interactions parents-jeunes. En 1963, KUHME mettait au point un dispositif expérimental lui permettant de soumettre des parents d'Hemichromis bimaculatus à plusieurs effluents dont un issu d'un réservoir contenant leurs propres jeunes. L'auteur constatait que les parents s'orientaient vers ce dernier et arboraient un comportement parental typique (* ventilation *).

Utilisant une technique analogue, MYRBERG put mettre en évidence en 1966 un phènomène identique chez un autre Cichlidé, Cichlasoma nigrofasciatum. Cet auteur montra par ailleurs que la chémoréception jouait le rôle essentiel dans la préférence parentale des Cichlidés vis-à-vis des néonates, tandis que la vision interviendrait plus tard lorsque le jeune devient capable de nager en pleine eau.

Chez le mâle du Gourami bleu, Trichogaster trichopterus (Belontiidæ) au comportement parental de type voisin de celui des Cichlidés, KRAMER et LILEY montraient en 1971 que la réalisation de l'acte reproducteur inhibait sa tendance à dévorer les œufs.

\section{D) Reconnaissance intraspécifique et comportement de banc}

En 1932, WREDE montrait que le Vairon européen, Phoxinus phoxinus L., placé dans une situation de libre choix, marquait une préférence pour le compartiment de l'aquarium contenant l'odeur d'autres poissons de la même espèce.

BARDACH et TODD menèrent en 1970 une étude approfondie du rôle de l'olfaction dans la hiérarchie sociale du poisson chat Ictalurus natalis. Les auteurs montrèrent que les individus ètaient capables de percevoir le statut social de conspécifiques en l'absence de stimuli visuels. Ainsi, de l'eau émise d'un aquarium ayant contenu un dominé déclenchait chez un dominant un comportement d'attaque dans la zone de l'effluent. Si ce même dominant perdait son avantage hiérarchique à la suite d'interrelations agressives, le comportement d'attaque vis-à-vis de l'effluent n'avait pas lieu. Cette discrimination sociale disparaissait après lésions olfactives. D'apres ces auteurs et RICHARDS (1974), le mucus et !urine constitueraient les principaux agents responsables de la communication intraspécifique chez Ictalurus natalis.

Chez l'Omble chevalier, Salvelinus alpinus, HOGLUND (1973) mettait en évidence chez les juvéniles une forte attraction intraspécifique disparaissant après cautérisation de l'épithélium olfactif. D'autres auteurs tels que KEENLEYSIDE (1955), STEVENS (1959), KÜHME (1964) et HEMMINGS (1966) soutiennent ègalement l'hypothèse d'une participation importante de l'olfaction dans la formation 
et le maintien du banc. Selon HEMMINGS (1966) la cohésion du banc de gardans, Rutilus rutilus serait assuree principalement par la vision du jour, tandis que l'olfaction maintiendrait l'attraction interindividuelle intraspécifique de nuit.

\section{- Réaction d'alarme}

Une très abondante littérature traitant ce sujet s'est accumulèe depuis 1938 , date à laquelle VON FRISCH constatait qu'un vairon blessé provoquait la fuite de conspécifiques. VON FRISCH montra par la suite que la réaction d'alarme ( schreckreaction") était due à la perception d'une substance ("schreckstoff") émise à partir de la zone lésée de l'épiderme et qu'elle se manifestait chez de nombreuses espèces de cyprinidés.

L'extrait épidermique est actif à des doses infinitesimales puisque SCHUTZ (1956) rapporte des expériences menées chez le vairon dans lesquelles une dose de $140 \mathrm{pg} / \mathrm{ml}$ suffit à déclencher la réaction caractéristique. $\mathrm{r}_{1}$ 1960, PFEIFFER identifiait un type de cellules épidermiques particulières aux poissons susceptibles de présenter une réaction d'alarme. Ce type de cellules, à l'aspect en massue, ne peut relâcher son contenu qu'en cas de lésion. Après réalisation d'extraits épidermiques à partir de différentes zones du corps du poisson chat, Kriptopterus bicirrhis, PFEIFFER constatait que seuls les extraits réalisés à partir de zones présentant ces cellules (c'est-à-dire tout le corps à l'exception des barbillons) étaient susceptibles de déclencher une réaction d'alarme. D'après les travaux visant à définir la nature chimique de la substance d'alarme (HUTTEL, 1941 ; HUTTEL et SPRENGLING, 1943 ; PFEIFFER et LEMKE, 1973, LEBEDEVA et al., 1974), ii ressort que celle-ci appartiendrait au groupe des ptérines.

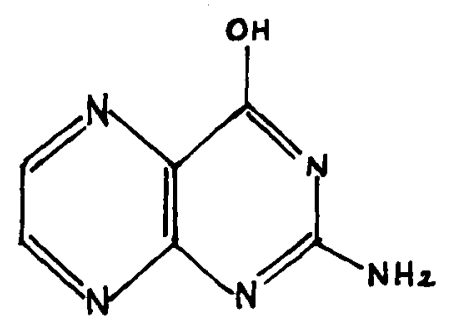

\section{Ptérine (Amino-2 OH-4 ptéridine)}

Cependant, quoique ce composé puisse effectivement faire apparaitre une réaction d'alarme chez certains cyprinidés, l'intensité de la réaction demeure dans tous les cas inférieure à celle produite par l'extrait naturel. D'autre part, bien que ces ptérines soient concentrées au niveau des xanthophores particulièrement abondants au niveau dorsal, les extraits réalisés chez le vairon à partir de la peau dorsale ou ventrale font preuve d'une efficacité identique (VON FRISCH, 1941 ; PFEIFFER, 1963). Les expériences de lésions olfactives montrent que, si le sens olfactif est principalement impliqué dans la détection de la substance d'alarme, la vision interviendrait également comme moyen de transmission de la réaction (SCHUTZ, 1956).

Présentes le plus souvent chez des poissons sociaux et non prédateurs, la substance d'alarme et la réaction qui lui est liée joueraient avant tout un rôle protecteur, contribuant à la survie de l'espèce concernée. Toutefois, chez certains cyprinidés présentant cette particularité, les cellules spécialisées dispa- 
raissent au moment de la fraie. Ces poissons peuvent ainsi nettoyer une aire de reproduction en se frottant contre le substrat sans déclencher l'émission de la substance d'alarme qui ferait fuir l'éventuel partenaire sexuel. SMITH (1973) rapporte le cas des mâles de Ménés à grosse tête. Pimephales promelas, qui perdent ces cellules au printemps, au début de la saison de reproduction, pour ne le récupérer qu'à l'automne. L'auteur montra qu'un traitement avec de la testostérone réduisait le nombre de ces cellules caractéristiques.

\section{- Effet de groupe}

En 1968, MILES constatait que la présence de grand nombre de civelles dans un cours deau le rendait moins attractif aux autres civelles migratrices. Les experiences de confinement de ROSE (1959) sur Tanichthys albonubes, Barbus tetrazona et Poecilia reticulata, ont montré l'effet négatif de fortes densités sur la fécondité des femelles, la croissance et le taux de survie des alevins. L'auteur montra que le sens chimique ètait principalement responsable de cet état de fait, dans la mesure où un apport constant d'eau pure ou un renouvellement d'eau plusieurs fois par jour suffisait pour produire une très nette amélioration de la situation.

YU et PERLMUTTER (1970) examinerent les facteurs d'inhibition de croissance chez Brachydanio rerio. Après passage de l'eau contenant une très forte population de Brachydanio rerio au travers un filtre à charbon. les auteurs constituèrent à partir du support absorbant un extrait capable de retarder le développement de ces poissons et de diminuer leur rythme cardiaque. Si l'on place deux lots identiques de ces poissons dans des aquariums qui se différencient par le seul fait que l'un est muni d'un filtre susceptible de collecter ces substances et l'autre pas, on constate une mortalité beaucoup plus importante dans l'aquarium non muni d'un filtre.

L'extrait isole par YU et PERLMUTTER sur Brachydanio rerio ne possédant aucun effet sur Trichogaster trichopterus ni sur Rivulus harti, les auteurs lui attribuèrent une certaine spécificité.

GREENE (1970) mit également en évidence chez Carassius auratus et chez d'autres espèces de cyprinidés, l'existence d'un facteur chimique capable d'inhiber la fraie. Lorsque l'auteur présentait à une faible population de poissons prêts à frayer de l'eau ayant contenu une très forte population de la même espèce, cela bloquait la fraie des premiers et, à l'inverse, si GREENE déversait de l'eau pure dans un aquarium contenant une très forte population de ces poissons, ces derniers se mettaient à frayer. Ceci doit être mis en relation avec les observations de LIN (1935) sur la carpe Cténopharyngodon idelus et de TANG (1957, 1963) sur la carpe commune Cyprinus carpio et sur la carpe argentée Hypophthalmichthys molitrix qui constataient que la fraie survenait chez ces espèces aussitôt après de fortes pluies.

D'autres auteurs cités par CHEN et MARTINICH (1975), tels que VON IHERING et WRIGHT (1955) et LAKE (1967), ont également reconnu l'importance de la relation existant entre l'apport d'eau (pluie et eaux de ruissellement) et "'nduction de la fraie chez les cyprinides.

En 1974, PFUDERER et al. tenterent d'identifier la nature chimique de ce facteur ou "crowding factor " chez Carassius auratus en fixant pour essai biologique son action sur la croissance et sur le rythme cardiaque. Les auteurs conciurent de leurs analyses physico-chimiques que les substances responsables pourraient être constituées de composés analogues à des esters de phtaiates combinés à d'autres constituants lipidiques. 


\section{III. - CONCLUSIONS ET PERSPECTIVES D'AVENIR}

Grâce à unn engagement pluridisciplinaire impliquant l'utilisation concertée de techniques d'études aussi variées que la psychologie sensorielle comparée. la neuroendocrinologie, l'histologie, l'électrophysiologie et l'analyse physicochimique, les dix dernières années de recherches dans le domaine des phéromones de poissons ont permis d'approfondir et d'élargir des connaissances restées longtemps fragmentaires.

Considérant le rôle primordial joué par ces médiateurs chimiques d'action spécifique dans les phénomènes de reproduction, de migration et de régulation des densités de population, on peut prévoir d'ores et déjà un certain nombre d'applications de valeur agro-économique non négligeable.

- La connaissance chimique de substances susceptibles de provoquer lattraction d'especes d'intérêt économique (ou écologique) pourrait être utilisée à des fins de contrôle de la présence éventuelle d'une population donnée dans uise zone déterminée;

- Il est également possible, après avoir mesuré l'impact éco-éthologique que peut produire l'introduction d'une nouvelle espèce dans la zone choisie, d'utiliser de telles substances attractives dians un but de repeuplement (cas de l'induction du "homing " chez les salmonides) ou encore, et en dernière extrémitè ( $v u$ les dommages que cela serait susceptible d'occasionner sur les niveaux de populations), de pêche (cas de pêcheurs du Mississipi qui attirent les mâles d'ictalurus nebulosus en utilisant les femelles gravides comme leurre spécifique).

- Dans le cadre de la pisciculture où les densités de poissons excèdent le plus souvent celles existant dans le milieu naturel, l'existence de facteurs biochimiques (ou "crowding factors") susceptibles de diminuer la productivité doit être également prise en consideration. Ceci est particulièrement vrai dans le cas des installations fonctionnant en circuit fermé.

Toujours dans l'optique de l'amélioration de la productivité piscicole, l'utilisation de phéromones sexuelles synthétiques pourrait être enfin envisagée dans le but de synchroniser, sinon d'induire, la reproduction, surtout dans les cas où celle-ci est associée à des traitements neuro-hormonaux.

Dans l'ètat actuel des connaissances, le principal obstacle au développement rapide de ces applications réside dans la difficulté à résoudre les problèmes posés par l'analyse physico-chimique des extraits, que ceux-ci soient réalisés directement, à partir de tissus et organes, ou indirectement à partir d'un support absorbant, charbon ou autre

\section{BIBLIOGRAPHIE}

AMOURIQ L., 1965. Origine de la substance dynamogène èmise par Lebistes reticulatus femelle (Poisson Poeciliidae, Cyprinodonti forme)

C.R.Acad. Sci., Paris, 260, 2334-2335.

BARDACH J.E., TODD J.H., 1970. Chemical communication in Fish. In Advances in chemoreception. Vol. I. communication by Chemicals Signals (J.W. Johinson, D. G. Moulton and A. Turk, Eds.), pp. 205-240, Appleton. Century, Crofts, New York. 
BUCKLAND F., 1880. Natural history of British Fishes. Unwin London. p. 420

CARI_IN B., 1959. Results of salmon smolt tagging in the Baltic area. Rapp P.V. Reuit. Cons Int. Explor. Mer, 147, 89-96

CARLIN B., 1968. Salmon conservation, tagging experiments, and migrations of salmon in Sweden. Atl. Salmon Assoc., Montreal, Lect. Ser., pp. 22.

CHEN L.C., MARTINICH R.L., 1975 Pheromonal stimulation and metabolite inhibition of ovulation in the zebrafish, Brachydanio rerio. Fish. Bull., 73, 889-894.

COOPER J.C., SCHOLZ A.T., 1976. Homing of artificially imprinted steelhead (Rainbow) trout. Salmo gairdneri.

J. Fish. Res. Bd. Can., 33, 826-829

COOPER J.C., SCHOLZ A. T., HORRALL R.M., HASLER A.D., MADISON D.M., 1976. Experimental confirmation of the olfactory hypothesis with homing, artificially imprinted Coho salmon (Oncorhynchus kisutch) J. Fish. Res. Bd. Can., 33, 703-710.

CROW R.T., LILEY N.R., 1979. A sexual pheromone in the guppy. Poecilia reticulata (Peters). Can. J. Zool., 57, 184-188.

DODSON J.J., LEGGET W.C., 1974. Role of olfaction and vision in the behavior of american shad (Alosa sapidissima) homing to the Connecticut river from Long Island sound. J. Fish. Res. Bd. Can., 31, 1607-1609.

DONALDSON R., ALLEN G.H., 1957. Return of silver salmon Oncorhynchus kisutch (Walbaum) to point of release. Trans. Am. Fish. Soc., 87, 13-22.

EMANUEL M.E., DODSON J.J., 1979. Modification of the rheotropic behavior of male rainbow trout (Salmo gairdneri) by ovarian fluid.

J. Fish. Res. Bd. Can., 36, 63-68.

FRINGS H., FRINGS M., 1964. Animal communication. New York : Blaisdell Publishing $\mathrm{Co}$.

GERKING S.D., 1955. The restricted movements of fish populations. Biol. Rev., $34,221-242$.

GREENBERG B., 1963. Parental behaviour and recognition of young in Cichlasoma biocellatum. Anim. Behav., 11, 578-582.

GREENE G. N., 1970. A reproduction control factor in fishes. Ph. D. Dissertation. Auburn University, Auburn, Alabama.

HARA T.J., 1974. Is morpholine an effective olfactory stimulant in fish? J. Fish. Res. Bd. Can., 31, 1547-1550.

HARA T.J., LAW Y.M.C., HOBDEN B.R., 1973a. Comparison of the olfactary response to amino acids in rainbow trout, brook trout and whitefish. Comp. Biochem. Physiol., 45A, 969-977.

HASLER A.D., WISBY W.J., 1951. Discrimination of stream odors by fishes and relation to parent stream behavior. Am. Nat., 85, 223-238.

HEMMINGS C. C., 1966. Olfaction and vision in fish schooling. J. Exp. Biol., 45. 449-464. 
HOGI.UND L.B., ASTRAND M., 1973. Preferences among juvenile char (Salvelinus alpinus L.) to intraspecific odours and water currents studied with the fluviarium technique.

Rep. Inst. Freshwat. Res. Drottningholm, 53, 21-30.

HÜTTEL R, 1941. Die chemische Untersuchung des Schreckstoffes aus elritzenhaut. Naturwissenschaften, 29, 333-334

HUTTEL R., SPRINGLING G., 1943. Über Ichthyopterin, einen blau fluorescierenden stoff aus Fischhaut. Liebigs Ann., 554, 69-82

IDLER D.R., FAGERLUND U.H.M., MAYOH H., 1956. Olfactory perception in migrating salmon. I.L. Serine, a salmon repellent in mammalian skin. J. Gen. Physiol., 39, 889-892.

JENSEN A.L., DUNCAN R. N., 1971. Homing of transplanted coho salmon. Prog. Fish. Cult., 33, 216-218.

KARLSON P., LUSCHER M., 1959. "Pheromones": a new term for a class of biologically active substances. Nature, 183, 55-56.

KEENLEYSIDE M.H.A., 1955. Some aspects of the schooling of fish. Behaviour, 8, 183-248.

KRAMER D.L., LILEY N.R., 1971. The role of spawning behaviour and stimili from the eggs in the induction of a parental response in the blue gourami, Trichogaster trichopterus (Pisces, Belontiidae) Anim. Behav., 19, 87-92.

KUHME W., 1963. Chemische ausgelöste Brutpflege und Schwarmreaktionen bei Hemichromis bimaculatus (Pisces). Z. Tierpsychol., 20, 688-704.

KUHME W., 1964. Eine chemisch ausgelöste schwarreaktionen bei jungen Cichliden. Naturwissenschaften, 51, 120-121.

KUNZER P., 1964 Weitere versuche zur auslösung der nachfolgereaktion bei jungfishchen von Nannacara anomala (Cichlidae)

Naturwissenchaften, 51, 419-420.

LAKE J.S., 1967. Rearing experiments with five species of Australian freshwater fishes. I - Inducement to spawning.

Aust. J. Mar. Freshwater Res., 18, 137-153.

LAUMEN J., PERN U., BLUM V., 1973. Investigations on the function and hormonal regulation of the anal appendices in Blennius pavo (Risso) J. Exp. Zool., $190,47-56$

LEBEDEVA N.Y., MALYUKINA G.A., KASUMYAN A.O., 1974. The natural repellent in the skin of Cyprinids.

LIN S.Y., 1935. Life history of waan-ue, Ctenopharyngodon idellus (Cuv. et Val) Lingnan Sci. J., 14, 271-274.

MADISON D.M., SCHOLZ A.T., COOPER J.C., HORRALL R.M., HASLER A.D.,

DIZON A.E., 1973. I - Olfaction hypotheses and salmon migration : a synopsis of recent findings. Fish. Res. Bd. Can. Tech. Rep., 414, pp. 35.

MILES S.G., 1968. Rheotaxis of elvers of the American eel (Anguilla rostrata) in the laboratory to water from different streams in Nova Scotia. J. Fish. Res. Bd. Can., 25, 1591-1602. 
MYRBERG Jr. A.A., 1966. Parental recognition of young in Cichlid fishes. Anim. Behav., 14, 565-571.

NEWCOMBE C., HARTMAN G., 1973. Some chemical signals in the spawning behaviour of rainbow trout (salmo gairdneri). J. Fish Res. Bd. Can., 30, 995997.

NOBLE G.K., CURTIS B., 1939. The social behaviour of the jewel fish Hemichromis bimaculatus Gill. Am. Mus. nat. Hist., B, 76, 1-46.

PARTRIDGE B.L., LILEY N.R., STACEY N.E., 1976. The role of pheromones in the sexual behaviour of the goldfish. Anim. Behav., 24, 291-299.

PECK J.W., 1970. Straying and reproduction of coho salmon. Oncorhynchus kisutch, planted in a lake superior tributary. Trans. Am. Fish. Soc., 99, 591-595.

PFEIFFER W., 1960. Uber die Schreckreaktion bei fischen und die Herkunft des Schreckstoffes. Z. Vergleich Physiol., 43, 578-614.

PFEIFFER W., 1963. Alarm substances. Experienta, XIX, 113-168.

PFEIFFER W., LEMKE J., 1973. Untersuchungen zur Isolariung und Identifizierung des Schreckstoffes aus der Haut der Elritze, Phoxinus phoxinus (L.) J. Comp. Physiol., 82, 407-410.

PFUDERER P., WILLIAMS P., FRANCIS A.A., 1974. Partial purification of the crowding factor from Carassius auratus and Cyprinus carpio J. Exp. Zool., 187, 375-382.

RICHARDS I.S., 1974. Caudal neurosecretory system : possible role in pheromone production. J. Exp. Zool., 187, 405-408.

ROSE S.M , 1959. Population control in guppies. Amer. Mild Naturalist, 62, 474-481.

SCHOLZ A.T., HORRALL R.M., COOPER J.C., HASLER A.D., MADISON D.M.,

POFF R.S., DALY R.I., 1975. Artifical imprinting of salmon and trout in lake Michigan. Wis. Dep. Nat. Resour. Fish Manage, Rep. 80, pp. 46.

SCHUTZ F., 1956. Vergleichende Untersuchungen uber die Schreckreaktion bei Fischen und deren Verbreitung. Z. Verg!. Physiol., 38, 84-135.

SMITH R.J.F., 1973. Testosterone eliminates alarm substance in male fathead minnows. Can. J. Zool., 51, 875-876.

STEVENS D.M., 1959. Studies in the schooling behavior of fish. J. Exp. Biol., 36, 261-280.

TANG Y.A., 1957. The effect of the repressive factor on fish reproduction (In Chin.) China Fish Man, 57, 2-3.

TANG Y.A., 1963. The testicular development of the silver carp Hypoththalmichthys molitrix ( $C$. et $\mathrm{V}$.) in captivity in relation to the repressive effects of wastes from fishes. Jap. J. Ichthyol., 10, 24-27.

TAVOLGA W.N., 1956. Visual chemical and sound stimuli as cues in the sex discriminatory behevior of the gobiid fish, Bathygobius soporator. Zooligica (N.Y.), 41, 49-64. 
TIMMS A.M., KLEEREKOPER H., 1972. The locomotor responses of male Ictalurus punctatus, the Channel catfish, to a pheromone released by the ripe female of the species. Trans. Amer. Fish. Soc., 2, 302-310.

VON FRISCH K., 1938. Zur physiologie des Fisch-Schwarmes.

Naturwissenchaften, 26, 601-606.

VON FRISCH K., 1941. Uber einen schreckstoff der Fischaut und seine biologische Bedeutung. Z. Vergleich. Physiol., 29, 46-145.

VON IHERING R., WRIGHT S., 1935. Fisheries investigations in northeast Brazil. Trans. Am. Fish. Soc., 65, 267-271.

WHITE H.C., 1934. Some facts and theories concerning the Atlantic salmon. Trans. Am. Fish. Soc., 64, 360-362.

WREDE W.L., 1932. Versuche uber den Artduft der Elritzen.

Z. Verg!. Physiol., 17, 510-519.

YU M.L., PERLMUTTER A., 1970. Growth inhibiting factors in the zebrafish (Brachydanio rerio) and the blue gourami (Trichogaster trichopterus) Growth, 34, 153-175. 